\title{
Foraging Behavior and Differences in Host Plant Selection of Mustard Saw Fly, Athalia lugens proxima Klug. on Different Brassica Species
}

\author{
Wajid Hasan $^{1,2}$, Harikesh Singh ${ }^{3 *}$ and C.P. Singh ${ }^{1}$ \\ ${ }^{1}$ Department of Entomology, G. B. Pant University of Agriculture and Technology, \\ Pantnagar-263145, Uttarakhand, India \\ ${ }^{2}$ Krishi Vigyan Kendra, Jehanabad- 804408, Bihar \\ ${ }^{3}$ Department of Entomology, Goacher Mahavidyalya, Rampur Maniharan, \\ Saharanpur- 247120, U.P., India \\ *Corresponding author
}

\section{A B S T R A C T}

Keywords

Athalia proxima lugens, Foraging,

Host plant,

Brassica.

Article Info

Accepted:

26 June 2017

Available Online:

10 July 2017
The present investigation was undertaken to investigate the Foraging behavior and differences in host plant selection of mustard saw fly, Athalia lugens on different Brassica spp. The highest grubs population of $A$. proxima were recorded at $6.00 \mathrm{PM}$ (2.68 grubs per linear meter row of plants) followed by at 6.00 AM (2.55), 9.00 AM (2.07), 12.00 PM (1.06) and lowest at 3.00 PM (1.06) and among different Brassica species, Crambe abyssinica $(0.47)$ and E. sativa $(0.38)$ were more resistant to $A$. proxima lugens and $B$. campestris (2.75) and $B$. juncea (2.90) were more susceptible.

\section{Introduction}

The mustard saw fly Athalia proxima lugens Klug. Is reported a serious crop pest causing extensive damage to different cruciferous plants in India (Butani and Jotwani 1984; Singh et al., 2007). Reduction in seed yield due to saw fly may go upto 100 (Sachan 1990). It appears in the pest form in early stage of the crop stage i.e. October and November. Reduction in yield due to saw fly on an average is about 25 per cent but it may go up to 100 per cent.

Sawfly activity was mostly found during seedling stage of the crop (Choudhury and Pal 2006). Larvae feed on leaves in groups of 3-6 larvae during the morning and evening periods.

In day time the grubs are found hidden in leaves (Upadhyay 2010). Athalia lugens proxima grubs feed on leaves of only family Cruciferae (Srivastava and Singh 1997).

The present investigation was undertaken to investigate the Foraging behavior and differences in host plant selection of mustard saw fly, Athalia lugens on different Brassica spp on crop which help us to apply control measures. 


\section{Materials and Methods}

The seeds of different Brassica species were sown in the month of October on the field of Crop Research Centre (CRC) of G. B. Pant University of Agriculture and Technology, Pantnagar 263145, India and recommended agronomic practices were applied and it was replicated thrice.

The observations on incidence started from 23 day after sowing (DAS) on the grubs population of $A$. proxima per linear meter rows of test plants at $6.00 \mathrm{AM}, 9.00 \mathrm{AM}$, 12.00 PM, 3.00 PM and at 6.00 PM. Data analysis was done statistically.

\section{Results and Discussion}

The results revealed that the highest cumulative mean grubs population of $A$. proxima were recorded 4.65 per linear meter row of plants on B. campestris cv. PT-30 followed by 4.42 on $B$. juncea $\mathrm{cv}$. Varuna, 4.24 on $B$. nigra $\mathrm{cv}$. PBR-I, 3.76 on $B$. campestris cv. BSH-I, 2.33 on B. napus cv. Sheetal, 2.06 on $B$. carinata $\mathrm{cv}$. CCN-06-01, 2.00 on B. campestris cv.YST-151, 1.56 on B. alba cv. PSB-I, 0.33 on E. Sativa cv. T-27 and lowest 0.13 on Crambe abyssinica cv. CAI at 6.00 AM (Table 1).

At 9.00 AM it was highest on PBR-I (3.65) followed by Varuna (3.19), PT-30(3.18), BSH-I(2.88), CCN-06-01(2.21), Sheetal (2.15), YST-15l(1.49), PSB-I(1.01), T27(0.65), CAI (0.24) (Table 2).

At 12.00 PM the highest population was PBR-I (1.74) followed by PT-30(1.72), Varuna (1.46), BSH-I (1.36), YST-151 (1.13), Sheetal (1.06), CCN-06-01 (0.99), PSB-I (0.82), T-27 (0.21) and again lowest on CAI (0.08) (Table 3). At 3.00 PM (Table 4) the population of grub was highest on BSHI(0.96) followed by PBR-I(0.71), Varuna(0.67), YST-151(0.67), PT-30(0.63),
Sheetal(0.53), CCN-06-01(0.43), PSB-I(0.40), $\mathrm{T}-27(0.18), \mathrm{CAI}(0.05)$ whereas at $6.00 \mathrm{PM}$ the highest population was 4.78 per linier meter row of plants on Varuna followed by 4.37 on BSH-I, 4.13 on PBR-I, 3.58 on PT30, 2.81 on Sheetal, 2.35 on CCN-06-01,2.00 on YST-151, 1.24 on PSB-I, 1.04 on CAl and lowest 0.54 on E. sativa cv. T-2-7 (Table 5).

On the basis of grand total find out that the highest grubs' population of A. proxima was recorded at 6.00 PM (2.68) followed by at 6.00 AM (2.55 per linear meter row of plants), 9.00 AM (2.07), 12.00 PM (1.06) and lowest at 3.00 PM (1.06) (Fig. 1).

It also concluded that among different species of Rapeseed-mustard, Crambe abyssinica (0.47) and E. sativa (0.38) were more resistant to $A$. proxima and $B$. campestris (2.75) and B. juncea (2.90) were more susceptible.

Three Athalia sawflies [A. japonica (Klug), A. rosae ruficornis Jakovlev, and A. infumata (Marlatt)] chose microhabitats, the suitability of each host plant for female oviposition and larval growth was similar.

Because of the divergent preferences for microhabitats, the host plants that were suitable for all the three steps were restricted to different sets of plants among the sawflies: Cardamine for A.japonica, cultivated crucifers (Raphanus and Brassica) for A. rosae, and Rorippa for A. infumata.

These plants could be recognised as the respective primary host plants (Koukichi and Naota 2002).

Significant negative impact of crop age was observed by Singh et al., 2008. Larvae feed on leaves in groups of 3-6 larvae during the morning and evening periods. In day time the grubs are found hidden in leaves (Mathur and Upadhyay 2010). 
Table.1 Population of active grubs of Atahlia proxima on different Brassica species at 6:00 A.M.

\begin{tabular}{|c|c|c|c|c|c|c|c|c|c|c|c|c|c|}
\hline Brassica species & 23 DAS & 24 DAS & 25 DAS & 26 DAS & 27 DAS & $28 \mathrm{DAS}$ & 29 DAS & $30 \mathrm{DAS}$ & $31 \mathrm{DAS}$ & 32 DAS & 33 DAS & 34 DAS & Mean \\
\hline B. alba cv. PSB-I & $3.33(10.37)$ & $3.33(10.37)$ & $2.00(7.80)$ & $2.50(7.24)$ & $1.67(5.94)$ & $2.17(7.18)$ & $0.50(2.87)$ & $1.50(5.49)$ & $0.50(2.87)$ & $0.33(1.91)$ & $0.50(2.87)$ & $0.33(1.91)$ & $1.56(5.54)$ \\
\hline E. sativa cv.T-27 & $0.83(4.22)$ & $0.50(2.87)$ & $0.33(1.91)$ & $0.00(0.00)$ & $0.17(0.96)$ & $0.67(2.62)$ & $0.83(2.88)$ & $0.00(0.00)$ & $0.17(0.96)$ & $0.33(1.91)$ & $0.00(0.00)$ & $0.17(0.96)$ & $0.33(1.91)$ \\
\hline B. campestris cv. BSH-I & $11.17(17.83)$ & $9.00(17.13)$ & $5.50(13.49)$ & $7.50(15.82)$ & $6.33(14.36)$ & $1.83(5.27)$ & $1.17(4.93)$ & $1.17(4.93)$ & $0.83(4.78)$ & $0.50(2.87)$ & $0.00(0.00)$ & $0.17(0.96)$ & $3.76(8.53)$ \\
\hline B. napus cv. Sheetal & $5.67(13.69)$ & $4.83(12.50)$ & $3.67(10.56)$ & $4.17(9.49)$ & $2.50(7.40)$ & $1.50(6.93)$ & $1.67(5.58)$ & $2.33(8.43)$ & $0.67(3.27)$ & $1.17(4.93)$ & $0.17(0.96)$ & $0.17(0.96)$ & $2.33(7.05)$ \\
\hline B. carinata cv. CCN-06-01 & $5.83(13.81)$ & $4.17(11.62)$ & $3.17(9.97)$ & $2.67(8.45)$ & $3.33(9.20)$ & $2.33(8.69)$ & $1.33(5.98)$ & $0.83(4.22)$ & $0.83(4.22)$ & $0.17(0.96)$ & $0.00(0.00)$ & $0.00(0.00)$ & $2.06(6.38)$ \\
\hline B. juncea cv. Varuna & $14.83(22.56)$ & $11.67(19.64)$ & $7.67(15.61)$ & $4.83(12.51)$ & $6.00(14.04)$ & $3.33(9.34)$ & $2.00(6.60)$ & $1.67(5.02)$ & $0.67(2.71)$ & $0.50(2.87)$ & $0.33(1.91)$ & $0.00(0.00)$ & $4.42(9.40)$ \\
\hline B. nigara cv. PBR-I & $12.67(20.03)$ & $8.33(16.08)$ & $5.33(13.23)$ & $7.33(15.43)$ & $6.67(14.76)$ & $2.00(7.25)$ & $2.00(7.25)$ & $2.67(8.36)$ & $1.00(3.97)$ & $1.33(5.33)$ & $0.50(2.87)$ & $1.00(5.18)$ & $4.24(10.04)$ \\
\hline Crambe abyssinica cv. CA-I & $0.00(0.00)$ & $0.00(0.00)$ & $0.00(0.00)$ & $0.17(0.96)$ & $0.00(0.00)$ & $0.83(3.02)$ & $0.33(1.91)$ & $0.00(0.00)$ & $0.00(0.00)$ & $0.00(0.00)$ & $0.17(0.96)$ & $0.00(0.00)$ & $0.13(0.57)$ \\
\hline B. campestris cv. YST-151 & $5.17(12.87)$ & $4.17(11.64)$ & $2.83(9.48)$ & $4.17(11.52)$ & $2.83(9.35)$ & $0.17(0.96)$ & $0.67(3.27)$ & $1.00(4.62)$ & $0.67(3.27)$ & $1.50(6.84)$ & $0.50(2.87)$ & $0.33(1.91)$ & $2.00(6.55)$ \\
\hline B. campestris cv. PT- 30 & $11.33(19.49)$ & $7.83(16.04)$ & $8.00(15.97)$ & $12.83(20.42)$ & $3.83(11.17)$ & $3.33(8.41)$ & $3.33(9.99)$ & $3.33(10.32)$ & $1.17(5.57)$ & $0.83(4.78)$ & $0.00(0.00)$ & $0.00(0.00)$ & $4.65(10.18)$ \\
\hline \multirow[t]{2}{*}{ Mean } & $7.08(13.49)$ & $5.38(11.75)$ & $3.85(9.80)$ & $4.62(10.18)$ & $3.33(8.71)$ & $1.82(5.97)$ & $1.33(5.19)$ & $1.40(5.14)$ & $0.65(3.11)$ & $0.67(3.24)$ & $0.22(1.24)$ & $0.22(1.19)$ & $2.55(6.59)$ \\
\hline & \multicolumn{2}{|c|}{ Brassica species } & \multicolumn{2}{|c|}{ DAS } & \multicolumn{2}{|c|}{ Brassica species x DAS } & & & & & & & \\
\hline SEm \pm & \multicolumn{2}{|c|}{0.229} & \multicolumn{2}{|c|}{0.251} & \multicolumn{2}{|c|}{0.794} & & & & & & & \\
\hline $\mathrm{CD}$ at $5 \%$ & \multicolumn{2}{|c|}{0.637} & \multicolumn{2}{|c|}{0.698} & \multicolumn{2}{|c|}{2.206} & & & & & & & \\
\hline
\end{tabular}

Table.2 Population of active grubs of Atahlia proxima on different Brassica species at 9:00 A.M.

\begin{tabular}{|c|c|c|c|c|c|c|c|c|c|c|c|c|c|}
\hline Brassica species & 23 DAS & 24 DAS & 25 DAS & 26 DAS & 27 DAS & $28 \mathrm{DAS}$ & $29 \mathrm{DAS}$ & $30 \mathrm{DAS}$ & $31 \mathrm{DAS}$ & 32 DAS & 33 DAS & 34 DAS & Mean \\
\hline B. alba cv. PSB-I & $0.67(3.27)$ & $1.50(4.67)$ & $2.50(6.40)$ & $1.33(4.54)$ & $0.83(3.02)$ & $1.17(3.51)$ & $1.17(4.93)$ & $1.50(5.64)$ & $0.67(3.27)$ & $0.17(0.96)$ & $0.33(1.91)$ & $0.33(1.91)$ & $1.01(3.67)$ \\
\hline E. sativa cv.T-27 & $0.33(1.91)$ & $0.50(2.31)$ & $1.83(5.43)$ & $0.50(2.31)$ & $0.17(0.96)$ & $0.83(4.22)$ & $0.50(2.31)$ & $1.50(4.63)$ & $0.83(4.22)$ & $0.33(1.91)$ & $0.33(1.91)$ & $0.17(0.96)$ & $0.65(2.84)$ \\
\hline B. campestris cv. BSH-I & $3.00(9.48)$ & $7.33(15.31)$ & $6.00(13.83)$ & $5.67(13.41)$ & $5.17(12.82)$ & $1.83(6.94)$ & $1.33(4.54)$ & $1.33(5.75)$ & $1.00(4.33)$ & $1.17(5.58)$ & $0.50(2.87)$ & $0.17(0.96)$ & $2.88(8.00)$ \\
\hline B. napus cv. Sheetal & $2.67(8.36)$ & $5.83(13.57)$ & $5.00(12.43)$ & $3.17(9.97)$ & $1.67(6.68)$ & $0.83(3.67)$ & $1.17(4.93)$ & $2.67(9.09)$ & $1.83(7.55)$ & $0.83(3.67)$ & $0.17(0.96)$ & $0.00(0.00)$ & $2.15(6.74)$ \\
\hline B. carinata $\mathrm{cv} . \mathrm{CCN}-06-01$ & $3.50(10.49)$ & $4.33(11.57)$ & $5.00(12.49)$ & $3.33(10.29)$ & $3.50(10.24)$ & $0.83(3.58)$ & $2.17(8.35)$ & $1.67(6.59)$ & $0.67(2.62)$ & $1.00(4.62)$ & $0.50(2.87)$ & $0.00(0.00)$ & $2.21(6.98)$ \\
\hline B. juncea $\mathrm{cv}$. Varuna & $5.33(12.98)$ & $6.33(13.59)$ & $6.67(14.56)$ & $5.33(12.30)$ & $4.83(12.06)$ & $1.50(6.29)$ & $2.83(9.15)$ & $1.67(6.68)$ & $1.50(6.19)$ & $1.33(5.33)$ & $0.67(3.27)$ & $0.33(1.91)$ & $3.19(8.69)$ \\
\hline B. nigara cv. PBR-I & $6.17(13.55)$ & $5.17(12.40)$ & $7.17(14.82)$ & $6.83(14.96)$ & $6.33(14.25)$ & $2.17(6.69)$ & $3.67(9.54)$ & $2.50(8.05)$ & $1.00(4.62)$ & $1.33(5.98)$ & $1.00(4.62)$ & $0.50(2.87)$ & $3.65(9.36)$ \\
\hline Crambe abyssinica cv. CA-I & $0.00(0.00)$ & $0.17(0.96)$ & $0.33(1.36)$ & $0.17(0.96)$ & $0.00(0.00)$ & $0.00(0.00)$ & $0.33(1.91)$ & $1.00(3.97)$ & $0.17(0.96)$ & $0.33(1.91)$ & $0.00(0.00)$ & $0.33(1.91)$ & $0.24(1.11)$ \\
\hline B. campestris cv. YST-151 & $1.33(4.54)$ & $2.50(7.67)$ & $3.33(10.12)$ & $2.83(9.45)$ & $1.67(6.45)$ & $1.33(5.98)$ & $1.00(3.97)$ & $2.00(7.95)$ & $1.17(5.02)$ & $0.33(1.91)$ & $0.17(0.96)$ & $0.17(0.96)$ & $1.49(5.42)$ \\
\hline B. campestris cv. PT- 30 & $2.83(9.48)$ & $6.33(14.05)$ & $7.50(15.40)$ & $5.50(13.15)$ & $4.33(11.16)$ & $2.00(6.53)$ & $4.67(12.41)$ & $2.67(9.22)$ & $1.00(3.97)$ & $1.00(4.07)$ & $0.33(1.91)$ & $0.00(0.00)$ & $3.18(8.45)$ \\
\hline Mean & $2.58(7.41)$ & $4.00(9.61)$ & $4.53(10.68)$ & $3.47(9.14)$ & $2.85(7.77)$ & $1.25(4.74)$ & $1.88(6.15)$ & $1.85(6.86)$ & $0.98(4.29)$ & $0.78(3.59)$ & $0.40(2.13)$ & $0.20(1.15)$ & $2.07(6.13)$ \\
\hline & \multicolumn{2}{|c|}{ Brassica species } & \multicolumn{2}{|c|}{ DAS } & \multicolumn{2}{|c|}{ Brassica species x DAS } & & & & & & & \\
\hline SEm \pm & \multicolumn{2}{|c|}{0.219} & \multicolumn{2}{|c|}{0.20} & \multicolumn{2}{|c|}{0.759} & & & & & & & \\
\hline $\mathrm{CD}$ at $5 \%$ & \multicolumn{2}{|c|}{0.609} & \multicolumn{2}{|c|}{0.667} & \multicolumn{2}{|c|}{2.110} & & & & & & & \\
\hline
\end{tabular}


Table.3 Population of active grubs of Atahlia proxima on different Brassica species at 12:00 P.M.

\begin{tabular}{|c|c|c|c|c|c|c|c|c|c|c|c|c|c|}
\hline Brassica species & 23 DAS & 24 DAS & 25 DAS & 26 DAS & 27 DAS & $28 \mathrm{DAS}$ & 29 DAS & 30 DAS & $31 \mathrm{DAS}$ & 32 DAS & 33 DAS & 34 DAS & Mean \\
\hline B. alba cv. PSB-I & $1.50(5.64)$ & $1.83(5.94)$ & $1.17(3.51)$ & $1.00(3.97)$ & $2.33(7.09)$ & $0.50(2.87)$ & $0.83(2.88)$ & $0.17(0.96)$ & $0.17(0.96)$ & $0.00(0.00)$ & $0.17(0.96)$ & $0.17(0.96)$ & $0.82(2.98)$ \\
\hline E. sativa cv.T-27 & $0.17(0.96)$ & $0.00(0.00)$ & $1.67(5.89)$ & $0.17(0.96)$ & $0.17(0.96)$ & $0.00(0.00)$ & $0.00(0.00)$ & $0.00(0.00)$ & $0.00(0.00)$ & $0.33(1.91)$ & $0.00(0.00)$ & $0.00(0.00)$ & $0.21(0.89)$ \\
\hline B. campestris cv. BSH-I & $4.50(12.01)$ & $3.67(9.93)$ & $2.33(7.49)$ & $2.00(7.25)$ & $2.17(8.35)$ & $0.67(3.27)$ & $0.67(3.27)$ & $0.17(0.96)$ & $0.17(0.96)$ & $0.00(0.00)$ & $0.00(0.00)$ & $0.00(0.00)$ & $1.36(4.46)$ \\
\hline B. napus cv. Sheetal & $2.00(7.73)$ & $1.67(6.68)$ & $1.67(4.98)$ & $2.17(6.91)$ & $2.50(7.24)$ & $1.33(5.75)$ & $0.67(2.71)$ & $0.33(1.36)$ & $0.33(1.91)$ & $0.00(0.00)$ & $0.00(0.00)$ & $0.00(0.00)$ & $1.06(3.77)$ \\
\hline B. carinata cv. CCN-06-01 & $3.33(10.13)$ & $2.67(8.91)$ & $1.00(4.53)$ & $2.33(7.82)$ & $1.17(4.37)$ & $0.17(0.96)$ & $0.33(1.36)$ & $0.00(0.00)$ & $0.83(3.02)$ & $0.00(0.00)$ & $0.00(0.00)$ & $0.00(0.00)$ & $0.99(3.42)$ \\
\hline B. juncea cv. Varuna & $4.17(11.49)$ & $3.00(8.96)$ & $3.17(8.89)$ & $1.83(6.85)$ & $2.00(7.95)$ & $1.33(4.54)$ & $1.17(4.93)$ & $0.17(0.96)$ & $0.33(1.91)$ & $0.33(1.91)$ & $0.00(0.00)$ & $0.00(0.00)$ & $1.46(4.87)$ \\
\hline B. nigara cv. PBR-I & $3.17(9.11)$ & $4.00(11.22)$ & $3.17(10.01)$ & $3.00(9.80)$ & $1.83(6.99)$ & $1.50(6.29)$ & $0.67(2.71)$ & $1.17(3.59)$ & $0.50(2.31)$ & $0.50(1.66)$ & $0.83(3.02)$ & $0.50(2.31)$ & $1.74(5.75)$ \\
\hline Crambe abyssinica cv. CA-I & $0.00(0.00)$ & $0.00(0.00)$ & $0.33(1.91)$ & $0.00(0.00)$ & $0.00(0.00)$ & $0.00(0.00)$ & $0.50(1.66)$ & $0.00(0.00)$ & $0.00(0.00)$ & $0.00(0.00)$ & $0.17(0.96)$ & $0.00(0.00)$ & $0.08(0.38)$ \\
\hline B. campestris cv. YST-151 & $2.17(8.04)$ & $2.50(7.24)$ & $3.17(7.84)$ & $1.33(5.33)$ & $1.17(4.37)$ & $1.00(4.53)$ & $1.17(4.93)$ & $0.17(0.96)$ & $0.17(0.96)$ & $0.00(0.00)$ & $0.33(1.91)$ & $0.33(1.91)$ & $1.13(4.05)$ \\
\hline B. campestris cv. PT- 30 & $4.33(11.67)$ & $4.00(11.26)$ & $2.83(8.57)$ & $3.00(9.49)$ & $2.00(7.95)$ & $0.83(4.22)$ & $2.00(6.43)$ & $0.67(2.62)$ & $0.83(3.58)$ & $0.17(0.96)$ & $0.00(0.00)$ & $0.00(0.00)$ & $1.72(5.56)$ \\
\hline \multirow[t]{2}{*}{ Mean } & $2.53(7.68)$ & $2.33(7.01)$ & $2.05(6.36)$ & $1.68(5.83)$ & $1.53(5.58)$ & $0.73(3.24)$ & $0.80(3.09)$ & $0.28(1.14)$ & $0.33(1.91)$ & $0.13(0.64)$ & $0.15(0.68)$ & $0.10(0.52)$ & $1.06(3.61)$ \\
\hline & \multicolumn{2}{|c|}{ Brassica species } & \multicolumn{2}{|c|}{ DAS } & \multicolumn{2}{|c|}{ Brassica species x DAS } & & & & & & & \\
\hline SEm \pm & \multicolumn{2}{|c|}{0.148} & \multicolumn{2}{|c|}{0.162} & \multicolumn{2}{|c|}{0.513} & & & & & & & \\
\hline $\mathrm{CD}$ at $5 \%$ & \multicolumn{2}{|c|}{0.411} & \multicolumn{2}{|c|}{0.451} & \multicolumn{2}{|c|}{1.425} & & & & & & & \\
\hline
\end{tabular}

Table.4 Population of active grubs of Atahlia proxima on different Brassica species at 3:00 P.M.

\begin{tabular}{|c|c|c|c|c|c|c|c|c|c|c|c|c|c|}
\hline Brassica species & 23 DAS & 24 DAS & 25 DAS & 26 DAS & 27 DAS & 28 DAS & 29 DAS & $30 \mathrm{DAS}$ & 31 DAS & 32 DAS & 33 DAS & 34 DAS & Mean \\
\hline B. alba cv. PSB-I & $0.67(3.27)$ & $0.33(1.91)$ & $0.83(3.02)$ & $0.50(2.31)$ & $1.00(3.97)$ & $0.50(2.31)$ & $0.50(2.31)$ & $0.50(2.31)$ & $0.00(0.00)$ & $0.00(0.00)$ & $0.00(0.00)$ & $0.00(0.00)$ & $0.40(1.78)$ \\
\hline E. sativa cv.T-27 & $0.16(0.95)$ & $0.50(2.31)$ & $0.17(0.96)$ & $0.33(1.91)$ & $0.33(1.91)$ & $0.17(0.96)$ & $0.17(0.96)$ & $0.33(1.91)$ & $0.00(0.00)$ & $0.00(0.00)$ & $0.00(0.00)$ & $0.00(0.00)$ & $0.18(0.89)$ \\
\hline B. campestris cv. BSH-I & $1.83(7.64)$ & $1.67(6.59)$ & $1.83(6.99)$ & $1.33(5.33)$ & $2.17(8.35)$ & $1.17(4.93)$ & $0.67(3.27)$ & $0.17(0.96)$ & $0.17(0.96)$ & $0.33(1.91)$ & $0.17(0.96)$ & $0.00(0.00)$ & $0.96(3.94)$ \\
\hline B. napus cv. Sheetal & $1.00(5.18)$ & $0.67(3.27)$ & $1.00(5.18)$ & $0.83(3.67)$ & $0.83(3.67)$ & $1.00(3.97)$ & $0.33(1.91)$ & $0.50(2.31)$ & $0.17(0.96)$ & $0.00(0.00)$ & $0.00(0.00)$ & $0.00(0.00)$ & $0.53(2.51)$ \\
\hline B. carinata cv. CCN-06-01 & $1.17(5.57)$ & $0.67(3.27)$ & $0.33(1.91)$ & $1.00(4.62)$ & $0.50(2.31)$ & $0.50(2.31)$ & $0.00(0.00)$ & $0.17(0.96)$ & $0.33(1.91)$ & $0.17(0.96)$ & $0.33(1.91)$ & $0.00(0.00)$ & $0.43(2.15)$ \\
\hline B. juncea cv. Varuna & $1.67(7.24)$ & $1.17(5.58)$ & $0.67(3.27)$ & $0.67(3.27)$ & $0.83(3.67)$ & $1.17(4.93)$ & $0.83(3.67)$ & $0.50(2.86)$ & $0.33(1.91)$ & $0.17(0.96)$ & $0.00(0.00)$ & $0.00(0.00)$ & $0.67(3.16)$ \\
\hline B. nigara cv. PBR-I & $1.50(6.93)$ & $1.50(6.29)$ & $0.83(3.67)$ & $0.83(3.67)$ & $0.67(3.27)$ & $0.67(3.27)$ & $0.67(3.27)$ & $0.83(3.67)$ & $0.17(0.96)$ & $0.83(3.67)$ & $0.00(0.00)$ & $0.00(0.00)$ & $0.71(3.45)$ \\
\hline Crambe abyssinica cv. CA-I & $0.17(0.96)$ & $0.17(0.96)$ & $0.00(0.00)$ & $0.17(0.96)$ & $0.00(0.00)$ & $0.00(0.00)$ & $0.17(0.96)$ & $0.00(0.00)$ & $0.00(0.00)$ & $0.00(0.00)$ & $0.00(0.00)$ & $0.00(0.00)$ & $0.05(0.32)$ \\
\hline B. campestris cv. YST-151 & $1.17(5.58)$ & $1.50(6.29)$ & $1.33(5.89)$ & $0.83(3.67)$ & $1.00(4.62)$ & $0.33(1.91)$ & $1.00(4.62)$ & $0.33(1.91)$ & $0.17(0.96)$ & $0.00(0.00)$ & $0.00(0.00)$ & $0.00(0.00)$ & $0.67(3.16)$ \\
\hline B. campestris cv. PT- 30 & $0.83(3.67)$ & $1.00(4.62)$ & $1.50(6.28)$ & $1.50(6.28)$ & $0.83(3.67)$ & $0.83(3.67)$ & $0.17(0.96)$ & $0.33(1.91)$ & $0.17(0.96)$ & $0.00(0.00)$ & $0.00(0.00)$ & $0.00(0.00)$ & $0.63(3.01)$ \\
\hline Mean & $1.02(4.76)$ & $0.92(4.11)$ & $0.85(3.77)$ & $0.80(3.68)$ & $0.81(3.54)$ & $0.63(2.99)$ & $0.45(2.25)$ & $0.37(1.88)$ & $0.18(1.05)$ & $0.18(0.94)$ & $0.05(0.29)$ & $0.00(0.00)$ & $0.52(2.44)$ \\
\hline & \multicolumn{2}{|c|}{ Brassica species } & \multicolumn{2}{|c|}{ DAS } & \multicolumn{2}{|c|}{ Brassica species x DAS } & & & & & & & \\
\hline SEm \pm & \multicolumn{2}{|c|}{0.078} & \multicolumn{2}{|c|}{0.086} & \multicolumn{2}{|c|}{0.271} & & & & & & & \\
\hline $\mathrm{CD}$ at $5 \%$ & \multicolumn{2}{|c|}{0.217} & \multicolumn{2}{|c|}{0.238} & \multicolumn{2}{|c|}{0.754} & & & & & & & \\
\hline
\end{tabular}


Table.5 Population of active grubs of Atahlia proxima on different Brassica species at 6:00 P.M.

\begin{tabular}{|c|c|c|c|c|c|c|c|c|c|c|c|c|c|}
\hline Brassica species & 23 DAS & 24 DAS & 25 DAS & 26 DAS & 27 DAS & $28 \mathrm{DAS}$ & $29 \mathrm{DAS}$ & 30 DAS & $31 \mathrm{DAS}$ & 32 DAS & 33 DAS & 34 DAS & Mean \\
\hline B. alba cv. PSB-I & $4.83(12.37)$ & $2.83(8.62)$ & $2.17(7.55)$ & $2.50(8.13)$ & $0.33(1.91)$ & $0.33(1.91)$ & $0.33(1.91)$ & $0.67(3.27)$ & $0.17(0.96)$ & $0.33(1.91)$ & $0.17(0.96)$ & $0.17(0.96)$ & $1.24(4.11)$ \\
\hline E. sativa cv.T-27 & $1.17(4.93)$ & $1.00(3.97)$ & $0.50(2.31)$ & $0.83(3.67)$ & $0.83(3.67)$ & $1.00(3.97)$ & $0.67(2.61)$ & $0.33(1.91)$ & $0.00(0.00)$ & $0.17(0.96)$ & $0.00(0.00)$ & $0.00(0.00)$ & $0.54(2.28)$ \\
\hline B. campestris cv. BSH-I & $16.67(24.03)$ & $11.17(19.29)$ & $7.83(15.69)$ & $7.00(14.74)$ & $1.83(7.64)$ & $1.67(7.24)$ & $1.50(6.93)$ & $2.17(7.65)$ & $1.67(5.58)$ & $0.67(3.83)$ & $0.50(2.31)$ & $0.33(1.91)$ & $4.37(9.74)$ \\
\hline B. napus cv. Sheetal & $8.17(16.15)$ & $6.50(14.36)$ & $3.67(10.43)$ & $4.17(11.65)$ & $2.17(8.29)$ & $2.17(8.35)$ & $1.83(6.99)$ & $2.83(9.22)$ & $1.50(6.29)$ & $0.50(2.87)$ & $0.33(1.91)$ & $0.00(0.00)$ & $2.81(8.04)$ \\
\hline B. carinata cv. CCN-06-01 & $11.67(19.68)$ & $2.83(9.49)$ & $2.83(8.83)$ & $3.00(9.80)$ & $1.17(4.93)$ & $1.50(5.73)$ & $1.33(5.33)$ & $2.00(8.04)$ & $1.17(5.56)$ & $0.50(2.31)$ & $0.17(0.96)$ & $0.00(0.00)$ & $2.35(6.72)$ \\
\hline B. juncea $\mathrm{cv}$. Varuna & $15.83(23.09)$ & $8.33(16.27)$ & $10.00(17.82)$ & $10.50(18.46)$ & $3.33(9.25)$ & $2.50(8.09)$ & $2.00(7.95)$ & $2.50(8.74)$ & $0.83(3.67)$ & $1.00(4.62)$ & $0.33(1.91)$ & $0.17(0.96)$ & $4.78(10.12)$ \\
\hline B. nigara cv. PBR-I & $15.67(23.22)$ & $6.83(13.47)$ & $7.50(15.81)$ & $6.00(12.77)$ & $2.67(8.15)$ & $2.00(7.25)$ & $1.50(6.93)$ & $3.83(9.62)$ & $2.17(6.94)$ & $0.83(3.67)$ & $0.50(2.87)$ & $0.00(0.00)$ & $4.13(9.26)$ \\
\hline Crambe abyssinica cv. CA-I & $5.50(9.63)$ & $0.50(2.31)$ & $2.67(6.22)$ & $0.67(2.62)$ & $0.33(1.91)$ & $0.33(1.36)$ & $0.50(2.31)$ & $0.83(3.67)$ & $1.00(4.62)$ & $0.17(0.96)$ & $0.00(0.00)$ & $0.00(0.00)$ & $1.04(2.91)$ \\
\hline B. campestris cv. YST-151 & $6.33(14.42)$ & $2.67(9.17)$ & $3.00(9.61)$ & $3.83(11.11)$ & $1.83(7.64)$ & $1.67(7.33)$ & $1.33(5.98)$ & $1.67(6.68)$ & $0.50(2.31)$ & $0.67(3.83)$ & $0.33(1.91)$ & $0.17(0.96)$ & $2.00(6.70)$ \\
\hline B. campestris cv. PT- 30 & $11.50(19.71)$ & $9.17(17.13)$ & $6.67(14.23)$ & $6.67(14.41)$ & $2.67(9.31)$ & $2.17(8.35)$ & $1.33(5.89)$ & $1.33(5.19)$ & $0.50(2.31)$ & $0.67(3.83)$ & $0.17(0.96)$ & $0.17(0.96)$ & $3.58(8.52)$ \\
\hline Mean & $9.73(16.72)$ & $5.18(11.41)$ & $4.68(10.85)$ & $4.51(10.67)$ & $1.72(6.27)$ & $1.53(5.90)$ & $1.23(5.23)$ & $1.82(6.33)$ & $0.90(3.86)$ & $0.55(2.88)$ & $0.25(1.38)$ & $0.10(0.57)$ & $2.67(6.84)$ \\
\hline & \multicolumn{2}{|c|}{ Brassica species } & \multicolumn{2}{|c|}{ DAS } & \multicolumn{2}{|c|}{ Brassica species x DAS } & & & & & & & \\
\hline SEm \pm & \multicolumn{2}{|c|}{0.250} & \multicolumn{2}{|c|}{0.274} & \multicolumn{2}{|c|}{0.867} & & & & & & & \\
\hline $\mathrm{CD}$ at $5 \%$ & \multicolumn{2}{|c|}{0.695} & \multicolumn{2}{|c|}{0.761} & \multicolumn{2}{|c|}{2.407} & & & & & & & \\
\hline
\end{tabular}

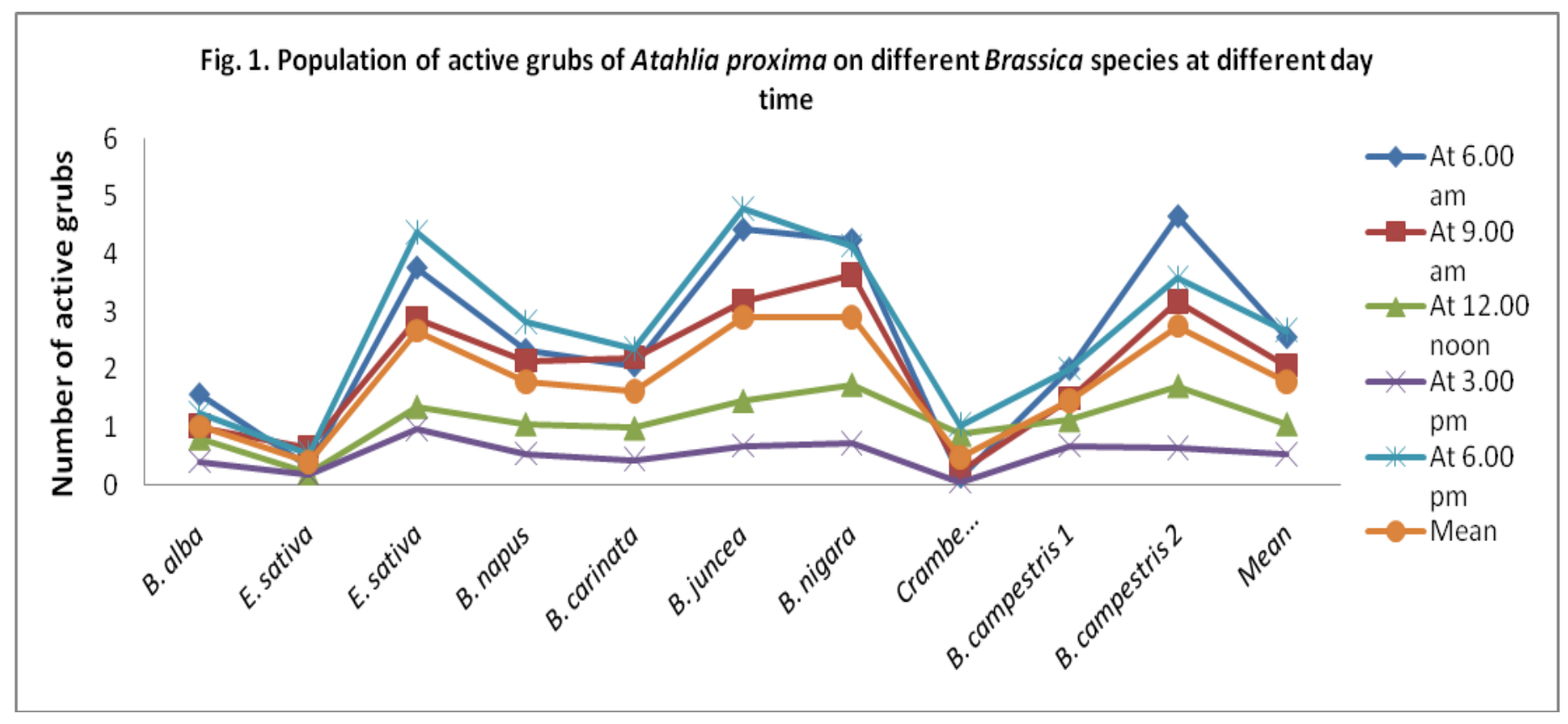




\section{References}

Choudhury, S and Pal, S. 2006. Pest complex and their succession in mustard under terai ecological conditions of West Bengal. Indian Journal of Entomology 68(4): 387-395.

Singh, R.K., Verma, R.A. and Sanjay Rajak 2008. Influence of environmental factors on the population fluctuations of mustard sawfly, Athalia proxima Klug on mustard crop. Environment and Ecology 26(3A): 1284-1286.

Butani, D.K. and Jotwani, M.G. 1984. Insect in vegetables. Periodical Exper Book Agency, New Delhi pp. 219-199.

Sachan, G.C. 1990. Insect pest problem in Brassicas and research work at Pantnagar II Brassica sub network meeting, GBPUA and T., Pannagar, 4-6 Jan 1988.

Koukichi Nagasaka and Naota Ohsaki 2002. Differences in host plant selection among three Athalia sawflies feeding on crucifers in Japan. Ecological Entomology 27(3) 326 - 336.

Singh, A.P., Singh, P.P. and Singh, Y. P. 2007. Pest complex of Indian mustard [Brassica juncea (L.) Czern and Cosson] in eastern Rajasthan. Journal of Experimental Zoology 10(2): 415-416.

Mathur Shukla, Prasad Upadhay (2010) Economic Zoology Biostatistics and Animal Behaviour. Rastogi Publication Meerut, India.

Srivastava, R.D. and Singh, R.P. 1997. An Introduction to Entomology. P 103.

\section{How to cite this article:}

Wajid Hasan, Harikesh Singh and Singh, C.P. 2017. Foraging Behavior and Differences in Host Plant Selection of Mustard Saw Fly, Athalia lugens proxima Klug. on Different Brassica Species. Int.J.Curr.Microbiol.App.Sci. 6(7): 2289-2294. doi: https://doi.org/10.20546/ijcmas.2017.607.329 